\title{
A NOTE ON THE THEOREM OF JARNIK-BESICOVITCH
}

\author{
by H. DICKINSON
}

(Received 19 April, 1996)

This note draws together and extends two recent results on Diophantine approximation and Hausdorff dimension. The first, by Hinokuma and Shiga [12], considers the oscillating error function $|\sin q| / q^{\tau}$ rather than the strictly decreasing function $q^{-\tau}$ of Jarnik's theorem. The second is Rynne's extension [17] to systems of linear forms of Borosh and Fraenkel's paper [3] on restricted Diophantine approximation with real numbers. Rynne's result will be extended to a class of general error functions and applied to obtain a more general form of [12] in which the error function is any positive function.

Before stating the problem some notation and definitions are introduced. Define $\|\mathbf{v}\|$ to be the distance of the nearest integer vector $\mathbf{p} \in \mathbb{Z}^{n}$ from $\mathbf{v} \in \mathbb{R}^{n}$, that is $\|\mathbf{v}\|=|\mathbf{v}-\mathbf{p}|_{\infty}$. Let $X$ be an $m \times n$ real matrix. Then

$$
\mathrm{a} X=\sum_{i=1}^{m} a_{i} x_{i j}, \quad j=1,2, \ldots, n,
$$

is a system of $n$ real linear forms in $m$ variables. The set of $\psi$ well-approximable $m \times n$ matrices is defined as

$$
W(m, n ; \psi)=\left\{X \in \mathbb{R}^{m n}:\|\mathbf{q} X\|<\psi(|\mathbf{q}|) \text { for infinitely many } \mathbf{q} \in \mathbb{Z}^{m}\right\} .
$$

This paper is concerned with the Hausdorff dimension of $W(m, n ; \psi)$ when instead of running over all $\mathbf{q}$ in $\mathbb{Z}^{m}$, we look at the $\mathbf{q}$ confined to a subset $Q \subseteq \mathbb{Z}^{m}$. Three applications of the main theorem will be given, one of which includes the case when $\psi$ is not necessarily a descreasing function. The set $W(m, n ; \psi)$ has been studied extensively; results about its Lebesgue measure can be found in $[16,10]$; about its Hausdorff dimension in $[14,15,1,8,2,7,5]$; and about its Hausdorff measure in $[15,4]$.

Define the unique number $0 \leq v(Q) \leq m$ such that

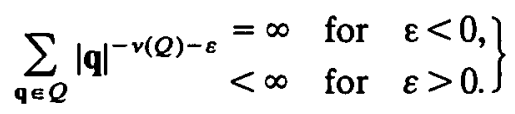

This is the exponent of convergence. From now on $W_{Q}(m, n ; \psi)$ will be used to denote the set

$$
W_{Q}(m, n ; \psi)=\left\{X \in \mathbb{R}^{m n}:\|\mathbf{q} X\|<\psi(|\mathbf{q}|) \text { for infinitely many } \mathbf{q} \in Q\right\} .
$$

Using this definition Rynne obtained the following result.

Theorem 1 (Rynne). For $\tau>v(Q) / n$

$$
\operatorname{dim} W_{Q}\left(m, n ; x^{-\tau}\right)=(m-1) n+\frac{v(Q)+n}{\tau+1} .
$$

Note that it follows from Groshev's theorem [10] that if $\tau<v(Q) / m$ then $W_{Q}$ has full Lebesgue measure.

As mentioned above this theorem extends the results of Borosh and Fraenkel [3],

Glasgow Math. J. 39 (1997) 233-236. 
who did the one dimensional case, and also of Eggleston [8] who considered certain subsets which were suitably sparse (corresponding to $v(Q)=0$ ) or suitably dense (corresponding to $v(Q)=1$ ). Bovey and Dodson [2] also looked at sequences which although "thin" were dense enough not to affect the Hausdorff dimension $(v(Q)=m)$. In particular they investigated a sequence of $\mathbf{q}$ whose coordinates were primes in arithmetic progressions; that is

$$
Q=\left\{\mathbf{q} \in \mathbb{Z}^{m}: q_{i} \equiv a_{i}\left(\bmod k_{i}\right), 1 \leq i \leq m\right\},
$$

where $\left(a_{i}, k_{i}\right)=1$.

With the objective of extending Rynne's result to a general class of error functions in mind, the lower and upper orders $\lambda$ and $\kappa$ of a function $f$ at infinity are defined respectively as

$$
\begin{aligned}
& \lambda(f)=\liminf _{r \rightarrow \infty} \frac{\log f(r)}{\log r}, \\
& \kappa(f)=\limsup _{r \rightarrow \infty} \frac{\log f(r)}{\log r} .
\end{aligned}
$$

Using these definitions let $\lambda_{\psi}=\lambda(1 / \psi)$ and $\kappa_{\psi}=\kappa(1 / \psi)$. Then for any $\varepsilon>0$ there exists an $N_{o}$ such that for all $N>N_{o}$

giving

$$
N^{\lambda_{\psi}-\varepsilon} \leq 1 / \psi(N) \leq N^{\alpha_{\psi}+\varepsilon},
$$

$$
N^{-\kappa_{\psi}-\varepsilon} \leq \psi(N) \leq N^{-\lambda_{\psi}+\varepsilon} \text {. }
$$

Further information on upper and lower order can be found in Hayman [11].

THEOREM 2. Let $\psi$ be a positive function; then

$$
(m-1) n+\frac{v(Q)+n}{1+\kappa_{\psi}} \leq \operatorname{dim} W_{Q}(m, n ; \psi) \leq(m-1) n+\frac{v(Q)+n}{1+\lambda_{\psi}}
$$

for $\lambda_{\psi}>v(Q) / n$.

When $Q=\mathbb{Z}^{m}$, Dodson [5] proved that the Hausdorff dimension of $W(m, n ; \psi)$ was in fact equal to $(m-1) n+(m+n) /\left(1+\lambda_{\psi}\right)$ for $\lambda_{\psi}>m / n$. When the lower and upper orders are equal the exact dimension is obtained.

From the definitions of upper and lower order we have that

$$
W_{Q}\left(m, n ; x^{-\kappa_{\psi}-\varepsilon}\right) \subset W_{Q}(m, n ; \psi) \subset W_{Q}\left(m, n ; x^{-\lambda_{\psi}+\varepsilon}\right) .
$$

This proves the theorem.

Three applications of Theorem 2 will now be discussed.

Application 1. This concerns the set $W(m, n ; \psi)$ when $\psi$ is not strictly decreasing.

THEOREM 3. Let $\psi: \mathbb{R} \rightarrow \mathbb{R}^{+}, \varphi: \mathbb{R} \rightarrow \mathbb{R}^{+}$be functions with $\varphi$ decreasing. Let $Q$ be an infinite set of integer vectors contained in $\mathbb{Z}^{m}$ for which $\varphi(|\mathbf{q}|) \leq \psi(|\mathbf{q}|)$, for all $\mathbf{q} \in Q$. Then

$$
(m-1) n+\frac{v(Q)+n}{\kappa_{\varphi}+1} \leq \operatorname{dim} W(m, n ; \psi) \leq(m-1) n+\frac{m+n}{\lambda_{\psi}+1},
$$

where $\lambda_{\psi}=\lambda(1 / \psi)$ and $\kappa_{\varphi}=\kappa(1 / \varphi)$.

Note that there is no growth condition on $\psi$ but there is no interest in looking at the set when $\psi$ is increasing. When $\psi(|\mathbf{q}|)$ decreases for certain integers one can take 
$\varphi(|\mathbf{q}|)=\psi(|\mathbf{q}|)$ for $\mathbf{q} \in Q$. The upper bound is obtained from [5] and the lower bound comes from the lower bound estimate in Theorem 2 . Obviously $W_{Q}(m, n ; \varphi) \subseteq$ $W(m, n ; \psi)$ and thus any lower bound for the dimension of $W_{Q}(m, n ; \varphi)$ is also a lower bound for $\operatorname{dim} W(m, n ; \psi)$.

Hinokuma and Shiga [12] obtained the exact dimension for the case $m=n=1$ with $\psi(q)=|\sin q| / q^{\tau}$. Since then, in 1996 Hinokuma and Shiga [13] have proved the complete result for a general function $\psi$ for the case $m=n=1$. Their proof involved a calculation of the number of $q$ such that $|\sin q|>c$. Thus the subset $Q$ was the set of $q \in \mathbb{Z}$ such that $|\sin q|>c$ and $\varphi(q)=c / q^{\tau}$. Using the notation of this paper they proved that $v(Q)=1$. Plainly Theorem 3 contains Hinokuma and Shiga's result, and indeed extends it to higher dimensions (the lower and upper orders are the same in this case). Clearly it will also deal with any other suitable trigonometrical function.

Another example is the function $\psi(r)=\phi(r) / r^{\tau}$, where $\phi$ is the Euler function, and so $\psi$ has a very irregular behaviour. For prime numbers however $\psi(r)=(r-1) / r^{\tau}$ and strictly decreases on the set of primes. In this case although the prime numbers can be thought of as a "thin" set, $v(Q)=1$ and the upper and lower bounds for the Hausdorff dimension are equal. Hence

$$
\operatorname{dim} W(1,1, \psi)=\frac{2}{\tau} \quad(\tau>2)
$$

Application 2. The following set

$$
V(\psi)=\left\{(X, \boldsymbol{\alpha}) \in \mathbb{R}^{m n} \times \mathbb{R}^{m}:\|\mathbf{q} X-\boldsymbol{\alpha}\|<\psi(|\mathbf{q}|) \text { for infinitely many } \mathbf{q} \in \mathbb{Z}^{m}\right\}
$$

was considered by Dodson in [6] and its Hausdorff dimension obtained using a different method for $\psi$ decreasing. This set is an inhomogeneous version of $W(m, n ; \psi)$. Bounds for its Hausdorff dimension can be obtained by rewriting it as $W_{Q}(m, n ; \psi)$ with $Q=\left\{(\mathbf{q},-1) \in \mathbb{Z}^{m+1}: \mathbf{q} \in \mathbb{Z}^{m}\right\}$, and considering the cartesian product of $X$ and $\boldsymbol{\alpha}$ as a matrix in $\mathbb{R}^{(m+1) n}$ with $\alpha$ as the final column vector. For this $Q$ it is readily verified that $v(Q)=m$ and then Theorem 2 gives the result

$$
m n+\frac{m+n}{1+\kappa_{\psi}} \leq \operatorname{dim} V(\psi) \leq m n+\frac{m+n}{1+\lambda_{\psi}} .
$$

In the event that $\psi$ is decreasing the exact Hausdorff dimension can be determined. As $\psi$ is decreasing, from the definition of lower order there exists a sequence of arbitrarily large integers $N$ such that $\psi(N)>N^{-\lambda_{\psi}-\varepsilon}$. It also holds that

$$
\psi(q) \geq c q^{-\lambda_{\phi}-\varepsilon}
$$

for $q \in\left[1 / 2 N_{r}, N_{r}\right]$ and some constant $c$. Choose a sequence $\left\{N_{r}\right\}_{r}$ of the $N$ 's for which $N_{r+1} \geq 4 N_{r}$ and let

$$
Q=\left\{(\mathbf{q},-1) \in \mathbb{Z}^{m+1}:|\mathbf{q}| \in\left[1 / 2 N_{r}, N_{r}\right] \text { for some } r\right\}
$$

It is readily verified that $v(Q)=m$. Also

$$
W_{Q}\left(m, n ; c q^{-\lambda_{\psi}-\varepsilon}\right) \subset V(\psi)
$$

giving the exact dimension. This observation is due to Bryan Rynne.

Application 3. Consider a point $X$ which lies in $W(m, n ; \psi)$. Let $Q(x)$ be the set of $\mathbf{q} \in \mathbb{Z}^{m}$ for which $\|\mathbf{q} X\|<\psi(|\mathbf{q}|)$. In some recent work by Forrest [9] the following condition was required: there exist other points, $X^{\prime}$ say, which obey the same inequality 
for $\mathbf{q} \in Q(X)$. (In [9] only one dimension was needed.) Using Theorem 2 it can now be shown that there exist uncountably many such points. By Theorem 2

$$
\operatorname{dim} W_{Q(X)}(m, n ; \psi) \geq(m-1) n+\frac{n+v(Q(X))}{1+\kappa_{\psi}}>(m-1) n .
$$

Thus the set of $X \in \mathbb{R}^{m n}$ that approximate the same $\mathbf{q}$ in $\mathbb{Z}^{m}$ has positive Hausdorff dimension strictly greater than $(m-1) n$, which shows that there are uncountable many such points, and they do not all lie on the hyperplanes defined by $\mathbf{q} X=\mathbf{0}$.

ACKNowledgements. Thanks are due to Maurice Dodson for his help and for suggesting the problem; to the EPSRC and the SFB 170 (Göttingen) for financial support; and finally to Bryan Rynne for his very helpful comments.

\title{
REFERENCES
}

1. A. S. Besicovitch, Sets of fractional dimension (IV): On rational approximation to real numbers, J. London Math. Soc. 9 (1934), 126-131.

2. J. Bovey and M. M. Dodson, The Hausdorff dimension of systems of linear forms, Acta Arith. 45 (1986), 337-358.

3. I. Borosh and A. S. Fraenkel, A generalization of Jarnik's theorem on Diophantine approximations, Indag. Math. 34 (1972), 193-201.

4. H. Dickinson and S. Velani, Hausdorff dimension and linear forms, Preprint Mathematica Gottingensis, Heft 23 (1995). (J. Reine Angew. Math., to appear)

5. M. M. Dodson, Hausdorff dimension, lower order and Khintchine's theorem in metric Diophantine approximation, J. Reine Angew. Math. 432 (1992), 69-76.

6. M. M. Dodson, A note on metric inhomogeneous Diophantine approximation, J. Austral. Math. Soc., to appear.

7. M. M. Dodson, B. P. Rynne and J. Vickers, Diophantine approximation and a lower bound for Hausdorff dimension, Mathematika 37 (1990), 59-73.

8. H. G. Eggleston, Sets of fractional dimensions which occur in some problems of number theory, Proc. London Math. Soc. 54 (1951), 42-93.

9. A. H. Forrest, The limit points of certain classical exponential series and other continuous cocycles, Preprint.

10. A. V. Groshev, Un Théorème sur les systèmes des formes linéaires, Dokl. Akad. Nauk SSSR 19 (1938), 151-152.

11. W. K. Hayman, Multivalent functions (2nd Edition Cambridge University Press, 1994).

12. T. Hinokuma and H. Shiga, A remark on a Theorem of Jarník, Ryukyu Math. J. 5(1992), 1-6.

13. T. Hinokuma and $H$. Shiga, Hansdorff dimension of sets arising in Diophantine approximation, Kodai Math. J. 19 (1996) No. 3, 365-377.

14. V. Jarník, Diophantische Approximationen und Hausdorffsches Mass, Mat. Sb. 36 (1929), $371-382$.

15. V. Jarník, Über die simultanen diophantischen Approximationen, Math. Z. 33 (1931), 505-543.

16. A. Khintchine, Einige Sätze über Kettenbrüche, mit Anwendungen auf die Theorie der Diophantischen Approximationen, Math. Ann. 92 (1924), 115-125.

17. B. P. Rynne, The Hausdorff dimension of certain sets arising from Diophantine approximation by restricted sequences of integer vectors, Acta Arith., 61 (1992), 69-81.

\author{
Department of Mathematics \\ UNIVERSITY OF YORK \\ HESLINGTON \\ YORK YO1 5DD \\ ENGLAND
}

